EPJ manuscript No.

(will be inserted by the editor)

\title{
DVCS on nuclei: Observability and Consequences
}

\author{
A. Freund ${ }^{a}$ and M. Strikman ${ }^{b}$ \\ ${ }^{a}$ Institut für Theoretische Physik, Universität Regensburg, D-93040 Regensburg, Germany \\ ${ }^{b}$ Department of Physics, The Pennsylvania State University, University Park, PA 16802, USA
}

December 7, 2018

\begin{abstract}
In this paper, we discuss the feasibility of measuring deeply virtual Compton scattering (DVCS) on nuclei in a collider setting, as for example, the planned high-luminosity Electron-Ion-Collider (EIC). We demonstrate that employing our recent model for nuclear generalized parton distributions (nGPDs), the one-photon unpolarized DVCS cross section as well as the azimuthal- and spin asymmetry are of the same size as in the proton case. This will allow for an experimental extraction of nuclear GPDs with high precision shedding new light not only on nuclear shadowing at small $x_{b j}$ but also on the interplay of shadowing and nuclear enhancement at $x_{b j} \sim 0.1$.
\end{abstract}

PACS. 11.10.Hi 11.30.Ly 12.38.Bx

\section{Introduction}

Recent experimental studies of deeply virtual Compton scattering (DVCS) on protons at HERA 2,1] have reached a $x_{b j}, Q^{2}$ range where they now provide a sensitive test of current models of generalized parton distributions (GPDs) in nucleons 3.4 which encode the three dimensional structure of hadrons $5,6,7,8$. In particular, it was shown that the NLO predictions are very sensitive to the gluon GPD inside the proton 3.4 .

Recently, we performed 9] studies of nuclear GPDs starting with the nuclear parton densities modeled using the leading twist theory of nuclear shadowing (see [10] and references therein). We used the same approximations for connecting GPDs and forward parton densities as were used in the proton case in 4 which led to a good description of the current world DVCS data.

When applied to the calculation of nuclear DVCS amplitudes, we observed that the nuclear modifications were up to $50 \%$ larger for the imaginary part of the DVCS amplitude than for the equivalent inclusive case. This is due to the sensitivity of the imaginary part of the DVCS amplitude to larger longitudinal distances compared to the inclusive amplitude for the same $x_{b j}$ [5]. This enhanced sensitivity makes the imaginary part an ideal tool to study high gluon density effects like saturation in nuclear DVCS at values of $x_{b j}$ where these effects normally would not occur in inclusive reactions (see [5] for a detailed discussion). At the same time, we found a surprisingly large modification of the real part of the DVCS amplitude extending to the region of $x_{b j} \sim 0.1$, where nuclear effects for the quark distributions are very small. Technically, this occurred due to a delicate interplay between shadowing corrections for quarks and nuclear enhancements for gluons in this region (see 9 for details). This, however, implies that the real part of the nuclear DVCS scattering amplitude is extremely sensitive to different physical phenomena not present in a nucleon occurring at longitudinal distances which are orders of magnitude different (see for example [5] for a detailed discussion). In summary, nuclear DVCS observables will allow us to study the modification or deformation of the three dimensional distribution of particle correlations encode in nGPDs due to a nuclear environment. This will enable us to shed new light on the dynamical interplay of highly complex bound hadronic systems.

In this paper, we will address the question of the feasibility of experimentally testing our predictions of nuclear modifications for GPDs. The main difficulty inherent to coherent processes with nuclei is their being strongly dominated by scatterings at $-t \leq 3 / R_{A}^{2}$ with $R_{A}^{2}$ the mean square nuclear radius. Detection of the scattered nuclei in this case is impossible in collider kinematics and would be a very challenging task for a dedicated fixed target experiment. The current HERMES DVCS experiment 11 sums over the coherent and incoherent contributions with the incoherent term giving a significant contribution [12] (see also 13 for an extensive study of how coherent DVCS depends on the spin of the target as well as [14). At the same time, it is pretty straightforward for an experiment at the Electron-Ion Collider (EIC) planned at BNL 16] to select the process $e A \rightarrow e \gamma A$ to which both the QED Bethe-Heitler and DVCS process contribute.

In consequence, this paper will address the question what $t$-integrated observables can be used to study nuclear DVCS and, therefore, the physics of nuclear modifications to the three dimensional distribution of particle correlations in nuclei. 
A. Freund ${ }^{a}$ and M. Strikman ${ }^{b}$ : DVCS on nuclei: Observability and Consequences

It is worth emphasizing that the $t$-dependence of the DVCS amplitude for $200 \geq A \geq 16$ and small $x \leq 0.03$. $(200 / A)^{1 / 3}$ is predominatly given by the nuclear body form factor and hence it is not of particular interest. At the same time, knowing this $t$-dependence and using information about the $t$-integrated amplitudes for a range of nuclei will make it possible to reconstruct the DVCS amplitude as a function of the nuclear thickness, $T(b)=$ $\int_{-\infty}^{+\infty} \rho_{A}(z, b) d z$, hence reaching a nuclear optical thickness which is about 1.5 times larger than the average heavy nucleus thickness.

The paper is organized as follows. In Section 2 we summarize the necessary formulae for the relevant observables: the one-photon cross section, the azimuthal angle asymmetry (and equivalent charge asymmetry) due to the interference of DVCS and Bethe-Heitler amplitudes, as well as the single spin asymmetry due to the longitudinal polarization of the electron beam (the EIC design includes running with highly polarized electron beams). We will also discuss the issue of the $t$-dependence. In section 3 we give our numerical predictions for four spin zero nuclei (O16,Ca-40,Pd-110 and Pb-208) including kinematic twist-3 effects in leading order (LO) (a detailed QCD analysis of kinematic twist-3 effects on the nucleon for the EIC including QCD evolution was presented in [17). Since the LO twist-3 DVCS amplitudes, modulo the dynamic twist3 contributions, are totally expressible through twist-2 GPDs [18, the twist-3 nuclear DVCS amplitudes can be directly computed from the nuclear twist-2 GPDs we employ here. We demonstrate that it would be feasible to observe the single spin asymmetry at the EIC. The observation of the angular asymmetry, though it is large at the EIC, will require a very good energy and momentum resolution of the electron and photon detectors. An option for a positron source at the EIC would enable the easier measurement of the charge asymmetry containing complementary information to the azimuthal angle asymmetry. The observation of the DVCS contribution to the total cross section would require a measurement at the $10 \%$ accuracy level. It is also demonstrated that the twist- 3 effects in nuclei are much smaller than in the nucleon and completely negligible. We conclude with a summary where we argue that DVCS measurements with nuclei will be an important component of the EIC program in particular for the studies of the color transparency phenomenon in vector meson production.

\section{Summary of DVCS formulas}

The lepton level process, $l(k) A(P) \rightarrow l\left(k^{\prime}\right) A\left(P^{\prime}\right) \gamma\left(q^{\prime}\right)$, receives contributions from each of the graphs shown in Fig. 11. This means that the cross section will contain a pure DVCS-, a pure BH- and an interference term.

We choose to work in the target rest frame given in, for example, 15] (see Fig. 21), where the positive $z$-direction is chosen along the three-momentum of the incoming virtual photon. The incoming and outgoing lepton three-momenta form the lepton scattering plane, while the final state nucleus and outgoing real photon define the nucleus-photon
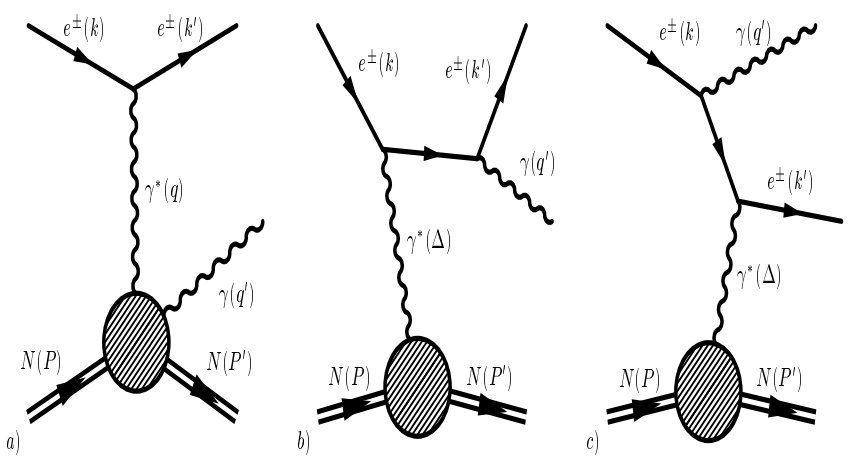

Fig. 1. a) DVCS graph, b) BH with photon from final state lepton and c) with photon from initial state lepton.

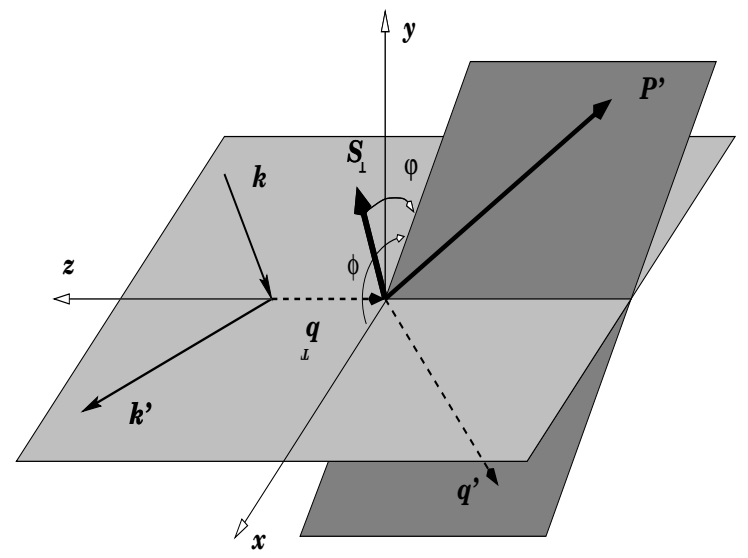

Fig. 2. The kinematics of the leptoproduction in the target rest frame.

scattering plane. In this reference frame the azimuthal angle of the scattered lepton is $\phi_{l}=0$, while the azimuthal angle between the lepton plane and the final state nucleus momentum is $\phi_{A}=\phi$. When the hadron is transversely polarized (within this frame of reference) $S_{\perp}=$ $(0, \cos \Phi, \sin \Phi, 0)$ and the angle between the polarization vector and the scattered hadron is given by $\varphi=\Phi-\phi_{A}$. The four vectors are $k=\left(E, E \sin \theta_{l}, 0, E \cos \theta_{l}\right), q=$ $\left(q^{0}, 0,0,-\left|q^{3}\right|\right)$. Other vectors are $P=\left(M_{A}, 0,0,0\right)$ and $P^{\prime}=\left(E_{A}^{\prime},\left|\boldsymbol{P}^{\prime}\right| \cos \phi \sin \theta_{A},\left|\boldsymbol{P}^{\prime}\right| \sin \phi \sin \theta_{A},\left|\boldsymbol{P}^{\prime}\right| \cos \theta_{A}\right)$. The longitudinal part of the polarization vector is $S_{\mathrm{LP}}=(0,0,0, \Lambda)$. The relevant Lorentz-invariant variables for DVCS are then:

$\xi_{A}=\frac{Q^{2}}{2 \bar{P} \cdot \bar{q}}, \overline{\mathcal{Q}}^{2}=-\bar{q}^{2}, t=\Delta^{2}=\left(P-P^{\prime}\right)^{2}, y=\frac{P \cdot q}{P \cdot k}$,

where $\bar{P}=\left(P+P^{\prime}\right) / 2, \bar{q}=\left(q+q^{\prime}\right) / 2$ and which are related to the experimentally accessible variables, $\zeta \equiv x_{b j}=$ $-A q^{2} / 2 P \cdot q=A \zeta_{A}$ and $Q^{2}=-q^{2}$, used throughout this paper, via

$$
\begin{aligned}
& \overline{\mathcal{Q}}^{2}=\frac{1}{2} Q^{2}\left(1+\frac{t}{Q^{2}}\right) \approx \frac{1}{2} Q^{2} \\
& \xi_{A}=\frac{\zeta_{A}\left(1+\frac{t}{2 Q^{2}}\right)}{2-\zeta_{A}\left(1-\frac{t}{Q^{2}}\right)} \approx \frac{\zeta}{2 A-\zeta} .
\end{aligned}
$$


Note that $t$ has a minimal value given by

$$
-t_{\min }^{2}=Q^{2} \frac{2 A\left(A-x_{b j}\right)\left(1-\sqrt{1+\epsilon^{2}}\right)+A^{2} \epsilon^{2}}{4 x_{b j}\left(A-x_{b j}\right)+A^{2} \epsilon^{2}} .
$$

where $\epsilon^{2}=4 M_{N}^{2} x_{b j}^{2} / Q^{2}$.

The corresponding five-fold differential cross section for a spin-0 target per nucleon is given by [15. to twist-3 accuracy neglecting transversity contributions:

$$
\frac{1}{A} \frac{d \sigma}{d x_{b j} d y d|t| d \phi d \varphi}=\frac{\alpha^{3} x_{b j} y}{16 \pi^{2} Q^{2} \sqrt{1+\epsilon^{2}} A^{3}}\left|\frac{\mathcal{T}}{e^{3}}\right|^{2} .
$$

Note that we give our nuclear amplitudes per nucleon [9]. In order to obtain the correct scaling of the cross section with $A$, the DVCS amplitude has to be multiplied by $A$. This leads to an extra factor of $A^{2}$ in the cross section which cancels two of the powers of $A$ in Eq. (3) to finally yield the correct overall power of $1 / A$.

The square of the amplitude receives contributions from pure DVCS (Fig. 1a), from pure BH (Figs. 1b, 1c) and from their interference (with a sign governed by the lepton charge),

$$
|\mathcal{T}|^{2}=\left|\mathcal{T}_{D V C S}\right|^{2}+\mathcal{I}+\left|\mathcal{T}_{B H}\right|^{2}
$$

where the individual terms are given by

$$
\begin{aligned}
& \left|\mathcal{T}_{\mathrm{BH}}\right|^{2}=-\frac{e^{6} F^{2}(t)}{x_{b j}^{2} y^{2}\left(1+\epsilon^{2}\right)^{2} t \mathcal{P}_{1}(\phi) \mathcal{P}_{2}(\phi)} \times \\
& {\left[c_{0}^{\mathrm{BH}}+\sum_{n=1}^{2} c_{n}^{\mathrm{BH}} \cos (n \phi)\right]} \\
& \left|\mathcal{T}_{\mathrm{DVCS}}\right|^{2}=\frac{e^{6}}{x_{b j} y^{2} \mathcal{Q}^{2}} \times \\
& {\left[c_{0}^{\mathrm{DVCS}}+\left[c_{1}^{\mathrm{DVCS}} \cos (\phi)+s_{1}^{\mathrm{DVCS}} \sin (\phi)\right]\right]} \\
& \mathcal{I}=\frac{ \pm e^{6} F(t)}{x_{b j}^{2} y^{3} t \mathcal{P}_{1}(\phi) \mathcal{P}_{2}(\phi)} \\
& {\left[\frac{t}{Q^{2}} c_{0}^{\mathcal{I}}+\sum_{n=1}^{2}\left[c_{n}^{\mathcal{I}} \cos (n \phi)+s_{n}^{\mathcal{I}} \sin (n \phi)\right]\right]}
\end{aligned}
$$

where the $+/-$ sign in the interference stands for a negatively/positively charged lepton and $F(t)$ is the nuclear form factor.

The $c_{n}$ 's and $s_{n}$ 's are the Fourier coefficients of the $\cos (n \phi)$ and $\sin (n \phi)$ terms. These coefficients are given as combinations of the real and imaginary part of the unpolarized DVCS amplitudes $\mathcal{H}$ (for the $c^{\mathcal{I}}$ 's or $s^{\mathcal{I}}$ 's) or the squares of the afore mentioned DVCS amplitude (for the $c^{\text {DVCS' }}$ or $s^{\text {DVCS' }}$ ). The exact form is given in [15] and does not have to be repeated here. The computation of the DVCS amplitudes and the necessary model assumptions were extensively discussed in 9] and will not be repeated here. The precise form of the $\mathrm{BH}$ propagators $\mathcal{P}_{1,2}(\phi)$ which induces an additional $\phi$-dependence, besides the $\cos (n \phi)$ and $\sin (n \phi)$ terms, and which can mock $\cos (n \phi)$ and $\sin (n \phi)$ dependences in certain observables, can also be found in [15]. Note that in order to avoid collinear singularities occurring through the coincidence of the outgoing photon with the incoming lepton line in $\mathcal{P}_{1,2}(\phi)$ we need to constrain $y$ according to

$$
y \leq y_{\mathrm{col}} \equiv \frac{Q^{2}+t}{Q^{2}+x_{b j} t} .
$$

in order to avoid an artificially enhanced $\mathrm{BH}$ contribution. This limit is only of practical relevance for fixed target experiments at very low energies. Collider experiments do not have any meaningful statistics for exclusive processes at very large $y \simeq O(1)$.

The DVCS observables we will discuss later on are based on a less differential cross section than the five-fold one in Eq. (3). The reason for this is first that the cross section in Eq. (3) is frame dependent since the azimuthal angles $\phi$ and $\varphi$ are not Lorentz invariants and hence, they will be integrated out. Secondly, since a $t$-distribution in nuclei will be impossible to measure, we also integrate out $t$, however, with experimentally sensible cuts as will be discussed later. In consequence, our observables will be based on only a two-fold differential cross section. In the following, we will concentrate on the Single Spin Asymmetry (SSA), the Charge Asymmetry (CA) and the azimuthal angle asymmetry (AAA) defined in accordance with experiments the following way:

$$
\begin{aligned}
& S S A=\frac{2 \int_{0}^{2 \pi} d \phi \sin (\phi)\left(d \sigma^{\uparrow}-d \sigma^{\downarrow}\right)}{\int_{0}^{2 \pi} d \phi\left(d \sigma^{\uparrow}+d \sigma^{\downarrow}\right)}, \\
& C A=\frac{2 \int_{0}^{2 \pi} d \phi \cos (\phi)\left(d \sigma^{+}-d \sigma^{-}\right)}{\int_{0}^{2 \pi} d \phi\left(d \sigma^{+}+d \sigma^{-}\right)}, \\
& A A A=\frac{\int_{-\pi / 2}^{\pi / 2} d \phi\left(d \sigma-d \sigma^{B H}\right)-\int_{\pi / 2}^{3 \pi / 2} d \phi\left(d \sigma-d \sigma^{B H}\right)}{\int_{0}^{2 \pi} d \phi d \sigma} .
\end{aligned}
$$

Here $d \sigma^{\uparrow}$ and $d \sigma^{\downarrow}$ refer to the two fold differential cross sections $d \sigma / d x_{b j} d Q^{2}$ with the lepton polarized along or against its direction of motion, respectively; $d \sigma^{+}$and $d \sigma^{-}$ are the unpolarized differential cross sections for positrons and electrons, respectively and $d \sigma^{B H}$ refers only to the pure BH cross section.

The EIC will most likely be running with electrons only and therefore the $\mathrm{CA}$ will not be measured. This leaves only the SSA and AAA. The experimental problem or challenge with the AAA, however, is that it requires either a very good detector resolution i.e. many bins in $\phi$ or an event by event reconstruction of the scattering planes. The last statement needs a word of explanation: Eq. (11) is equivalent to taking the difference between the number of DVCS minus BH events where the real $\gamma$ is above the electron scattering plane and where it is below that plane, divided by the total number of events. This procedure ensures that the numerator is not contaminated by $\mathrm{BH}$, which would spoil an unambiguous interpretation of the observable in terms of the real part of DVCS amplitudes. Also, the only difference between Eq. (10) and (11) 
is due to the additional interference term in the denominator of Eq. (11) and a twist- $2 \times$ twist- 3 contribution in the DVCS squared part which are both small in a collider setting compared with the leading contribution. Therefore, it does not matter from a physics point of view, whether one discusses the AAA or the $\mathrm{CA}$ !

We will also discuss the one-photon cross section $\sigma\left(\gamma^{*} A\right)$ at small $x_{b j}$ defined through

$$
\begin{gathered}
\frac{d^{2} \sigma(e A \rightarrow e A \gamma)}{d y d Q^{2}}=\Gamma \sigma_{D V C S}\left(\gamma^{*} A \rightarrow \gamma A\right) \\
\text { where } \quad \Gamma=\frac{\alpha_{e . m .}\left(1+(1-y)^{2}\right)}{2 \pi y Q^{2}} .
\end{gathered}
$$

with

$$
\frac{1}{A} \sigma_{D V C S}\left(\gamma^{*} A \rightarrow \gamma A\right)=\left.\frac{\alpha^{2} x_{b j}^{2} \pi}{Q^{4} \mathcal{B} A}\left|\mathcal{T}_{D V C S}\right|^{2}\right|_{t=0}
$$

and where $\mathcal{B}$ stems from the $t$-integration and will depend on both our cut-off in $t$ and the model of the $t$-dependence we will choose for the GPDs. Furthermore, all higher twist effects are neglected in this quantity.

Since we have already extensively discussed our nuclear GPD model in [9], we will not repeat this discussion here, except to remind the reader that it is based on our successful GPD parameterization for the nucleon [4] together with available nuclear shadowing parameterizations. For our discussion here, we will stick to the shadowing parameterization of [10] together with the CTEQ6 parameterization [19] as our necessary forward input distribution. Furthermore, we chose to model the nuclear $t$ dependence based on the parameterizations of the two component Fermi models employed in [10]. We also assume that the $t$-dependence factorizes from the GPD and thus also from the amplitude. This is, as in the case of the nucleon, not justified at all (see [17] for an extended discussion on this subject for the nucleon case). However, we are considering the cross section integrated over $t$, which is dominated by $-t \leq 3 / R_{A}^{2}$ i.e. $-t \leq 0.01 \mathrm{GeV}^{2}$ for all nuclei. In the case of small $x_{b j}$ we can use our experience of soft physics which indicates that rescattering effects change the slope of the amplitude by at most $5 \%$. For larger $x_{b j}$, it also seems natural to expect nonfactorizability effects to be small for small $t$. Hence, we expect this effect to be totally negligible, especially when compared to other factors like nucleus dissociation. Note also that though we perform all our calculations with a cut on our $t$ integration at $-t_{\max }=0.05 \mathrm{GeV}^{2}$, the contribution of $-t \geq-t_{\max }$ is negligible.

With the above discussion we have now all the tools in hand to estimate the sizes of nuclear DVCS observables at the EIC.

\section{Results for DVCS observables}

For the purposes of our discussion we choose to work with the maximal expected EIC setting for nuclei of $10 \mathrm{GeV}$ electrons and $100 \mathrm{GeV}$ nuclei. The range in $x_{b j}$ is between
$0.001-0.1$ and the $Q^{2}$ range is from $3-50 \mathrm{GeV}^{2}$ though the figures will only show the most advantageous kinematics which does not extend to the highest $Q^{2}$.

Since the EIC electron beam is expected to have polarization close to one, it would seem easiest to measure the SSA which directly probes the imaginary part of the DVCS amplitude. One can see from Figs. [3 - 6 that we predict the asymmetry to be of the order of $10 \%$ in a wide kinematic range which is roughly $25-50 \%$ less than in the nucleon case and nicely demonstrates the nuclear shadowing effects in the imaginary part of the DVCS amplitude. As a note on the side, we would like to quote our number for the SSA for coherent DVCS integrated over $t$ for O-16 for the average HERMES kinematics of $<Q^{2}>=2.2 \mathrm{GeV}^{2},<x_{b j}>=0.09$. We find an SSA of -0.24 (LO) and -0.22 (NLO) for O-16 compared to the HERMES SSA for Neon of $-0.22 \pm 0.03 \pm 0.03[11$. Note, however, that the preliminary HERMES data on nuclear DVCS do contain a contribution from incoherent DVCS, since the missing mass cut of $M_{X} \leq 1.7 \mathrm{GeV}$ may not remove all incoherent contributions with associated pion production and it definitely does not remove the contribution of the nucleus break-up channel.

We also predict a large azimuthal angular asymmetry (AAA) for all considered nuclei, Figs. 7 - 10 though a measurement of this asymmetry maybe require a very good angular resolution. Note that the asymmetry is as large or even a bit larger than in the nucleon case (see [17). This is very surprising given the fact that we expect a very large suppression of the real part of the DVCS amplitude in nuclei compared to the nucleon [9]. The answer to this apparent conundrum is hidden in the details of the calculation. The numerator of the AAA receives mainly contributions from $c_{0}^{\mathcal{I}}$ and $c_{1}^{\mathcal{I}}$ in our nuclear GPD model for both the nucleon and nuclei. However, for the nucleon which is spin $1 / 2$, there is a relative minus sign between these two terms compared to the spin 0 case we need to consider here, where there is a plus sign! This compensates for the relative suppression as compared to the nucleon case. Furthermore, in the kinematics where DVCS dominates over $\mathrm{BH}$, the nuclear shadowing effects reduce the denominator relative to the nucleon case which also partially compensates for the strong suppression effect in the real part of the nuclear amplitude. The measurement of the CA would be more advantageous from an experimental point of view since it contains the same information as the AAA.

Notwithstanding the experimental difficulties, a measurement of either the AAA or the CA would enable us to gain valuable insight into how particle correlations i.e. the nGPDs with the three dimensional information content are modified by the nuclear environment on very different longitudinal distance scales. Furthermore, we want to emphasize here that the measurement of the real part would be especially interesting since it is sensitive to the energy behavior of the cross sections at smaller $x_{b j}$ than the ones allowed by EIC kinematics. Hence, it is more sensitive to the proximity of the saturation/black body limit (see discussion in [20]). 
As one can see from our calculation, the kinematic twist-3 effects are entirely negligible for the EIC (as is similarly the case for the nucleon [17) in both the SSA and AAA. Furthermore, due to the nuclear shadowing corrections for the gluon reducing is relative numerical importance in the NLO amplitude, the NLO corrections for the SSA and AAA are less than in the case of the nucleon.

The one-photon cross section of DVCS per nucleon strongly increases with $A$ even in the region where the nuclear shadowing effects are important, see Figs. 11 18. As can be seen from the figures, the relative LO to NLO correction is less compared to the nucleon case and in line with the observation made for the SSA and AAA. The main problem for the measurement of the inclusive DVCS cross section is the subtraction of the BH QED background which also increases with $\mathrm{A}$. The ratio, $R$, of the DVCS to BH cross section is above 1 for $x_{b j} \geq 0.007$ and $Q^{2}=3 \mathrm{GeV}^{2}$ and rapidly increases as $x_{b j}$ increases $\left(R \simeq 10^{4}\right.$ for $\left.x_{b j} \geq 0.1\right)$. This is due to the fact that at fixed $Q^{2}, y$ deceases as $x_{b j}$ increases and, hence, $\mathrm{BH}$ which is $\propto y^{2}$, dies out rapidly. As $Q^{2}$ increases the value of $x_{b j}$ where $R \geq 1$ increases as well since $y$ now increases. For example, at $Q^{2}=25 \mathrm{GeV}^{2}, R \geq 1$ for $x_{b j} \geq 0.06$. These statements are essentially independent of $A$ and imply a very broad kinematic window where DVCS dominates over $\mathrm{BH}$, making a measurement of $\sigma\left(\gamma^{*} A\right)$ on the $10 \%$ level after subtraction of the $\mathrm{BH}$ contribution very feasible. Hence, it would be possible to perform two independent measurements of the imaginary part of the amplitude in a rather wide range of $x_{b j}, Q^{2}$, since $\sigma\left(\gamma^{*} A\right)$ is dominated by the imaginary part of the nuclear DVCS amplitude.

\section{Conclusions}

We have demonstrated that experiments at the EIC will allow one to measure the imaginary part of the nuclear DVCS amplitude using two independent techniques, the SSA and $\sigma\left(\gamma^{*} A\right)$. We also predict a large azimuthal angle asymmetry which could be studied using a high resolution detector. Such experiments will enable us to obtain unique information about quark nuclear GPDs, through scaling violations, as well as about gluon nuclear GPDs. The study of the A-dependence of the DVCS amplitude will allow one to determine the nuclear modification of nuclear GPDs as a function of the nuclear thickness up to the thicknesses $\rho_{0} \sim 2 \cdot R_{A} \sim 14 \mathrm{fm}$ where $\rho_{0}$ is the average nuclear density. Furthermore, it will allow us to verify the predicted larger nuclear modifications of the nGPDs 9] i.e. the modification of the three dimensional distribution and their connection to leading twist nuclear shadowing in the respective forward nuclear parton densities. These forward densities will also be studied at the EIC. Verifying the expectations of large nuclear effects for the real part of the DVCS amplitude at $x \sim 0.1$ would require running EIC at lower energies (which is also necessary for the measurements of the longitudinal DIS cross section) such that $\mathrm{BH}$ will be the dominating contribution in the cross section in the whole kinematic range.
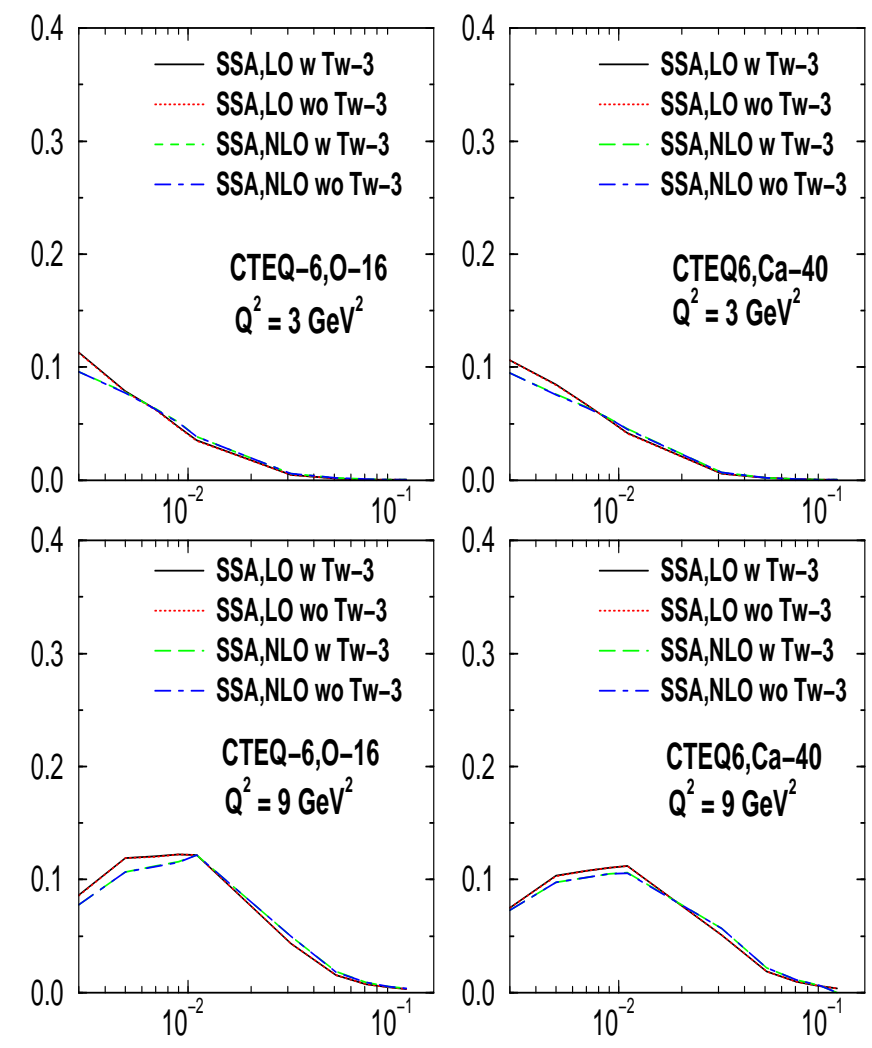

Fig. 3. $t$ integrated SSA in EIC kinematics vs. $x_{b j}$ for two typical values of $Q^{2}$ and $t_{\max }=-0.05 \mathrm{GeV}^{2}$. "W" stands for with and "WO" stands for without.
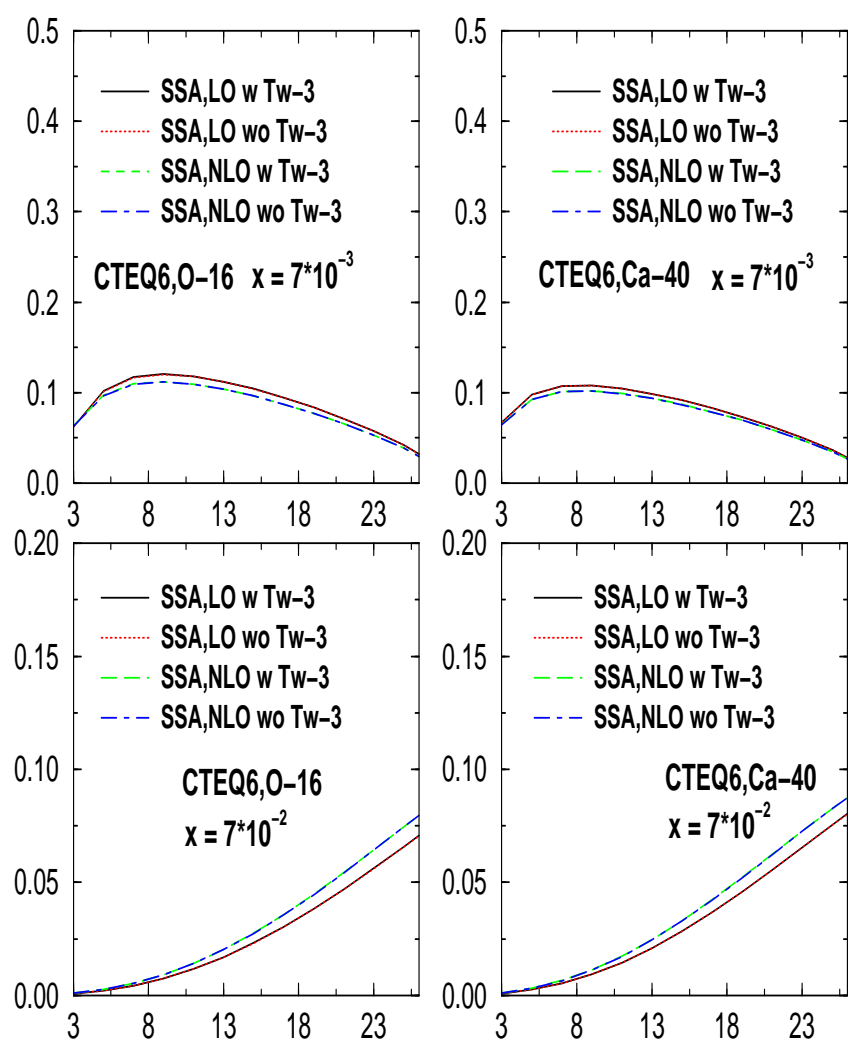

Fig. 4. $t$ integrated SSA in EIC kinematics vs. $Q^{2}$ for two typical values of $x_{b j}$ and $t_{\max }=-0.05 \mathrm{GeV}^{2}$. "W" stands for with and "WO" stands for without. 

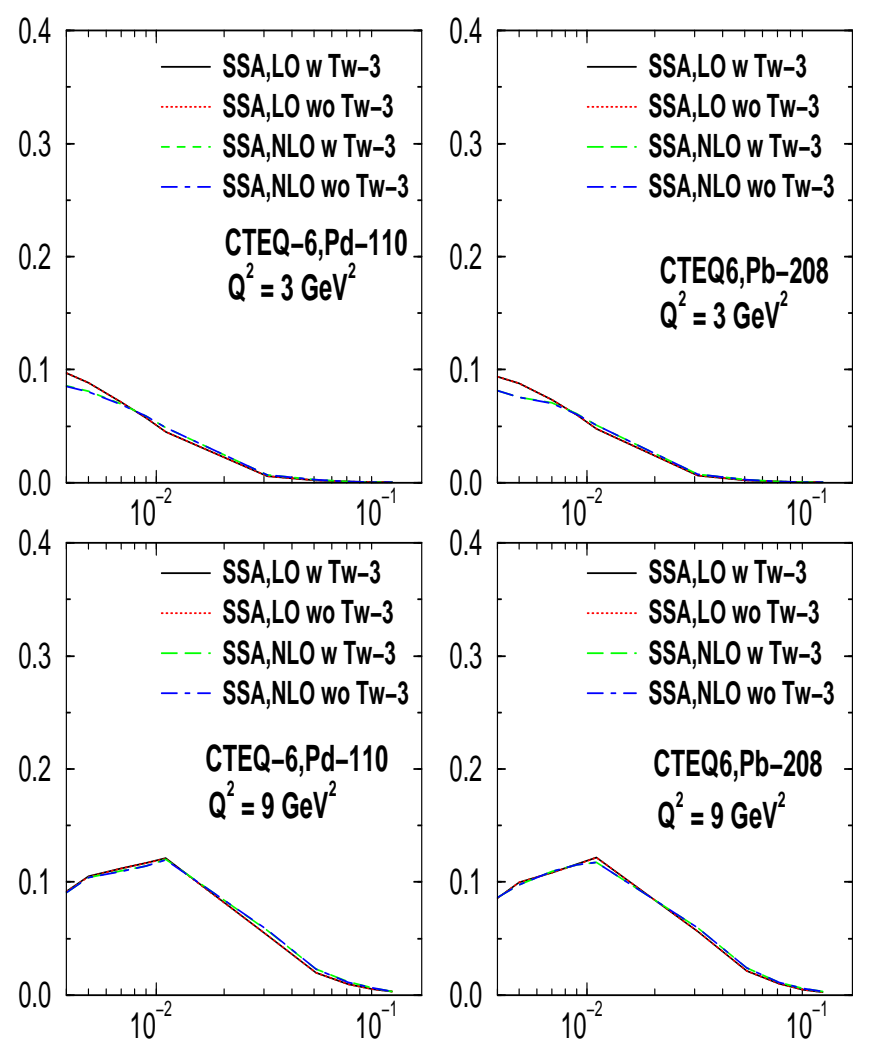

Fig. 5. $t$ integrated SSA in EIC kinematics vs. $x_{b j}$ for two typical values of $Q^{2}$ and $t_{\max }=-0.05 \mathrm{GeV}^{2}$. "W" stands for with and "WO" stands for without.
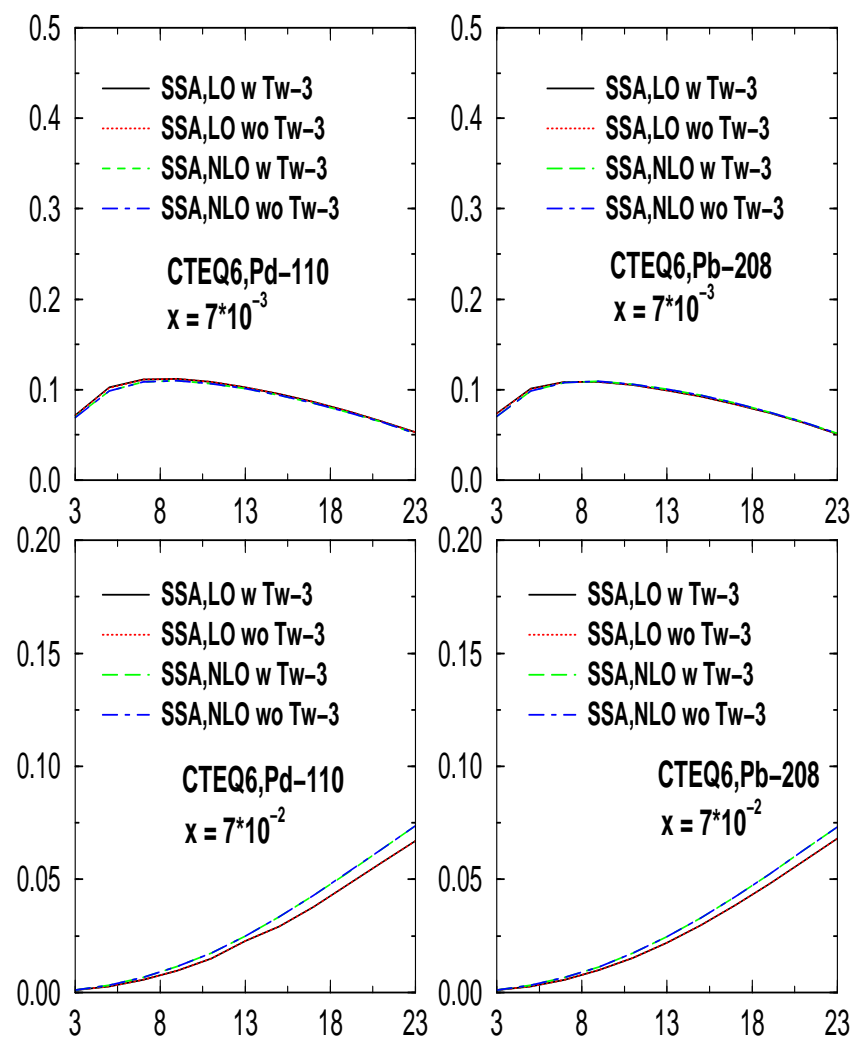

Fig. 6. $t$ integrated SSA in EIC kinematics vs. $Q^{2}$ for two typical values of $x_{b j}$ and $t_{\max }=-0.05 \mathrm{GeV}^{2}$. "W" stands for with and "WO" stands for without.
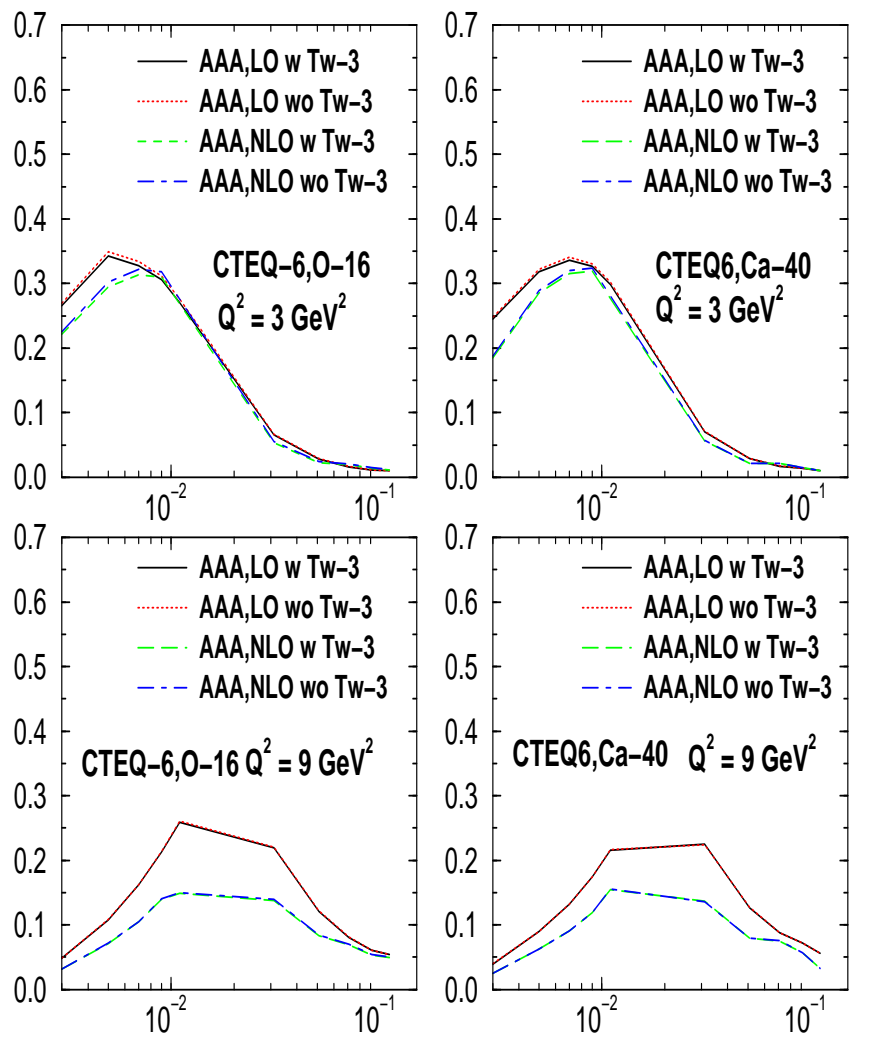

Fig. 7. $t$ integrated AAA in EIC kinematics vs. $x_{b j}$ for two typical values of $Q^{2}$ and $t_{\max }=-0.05 \mathrm{GeV}^{2}$."W" stands for with and "WO" stands for without.
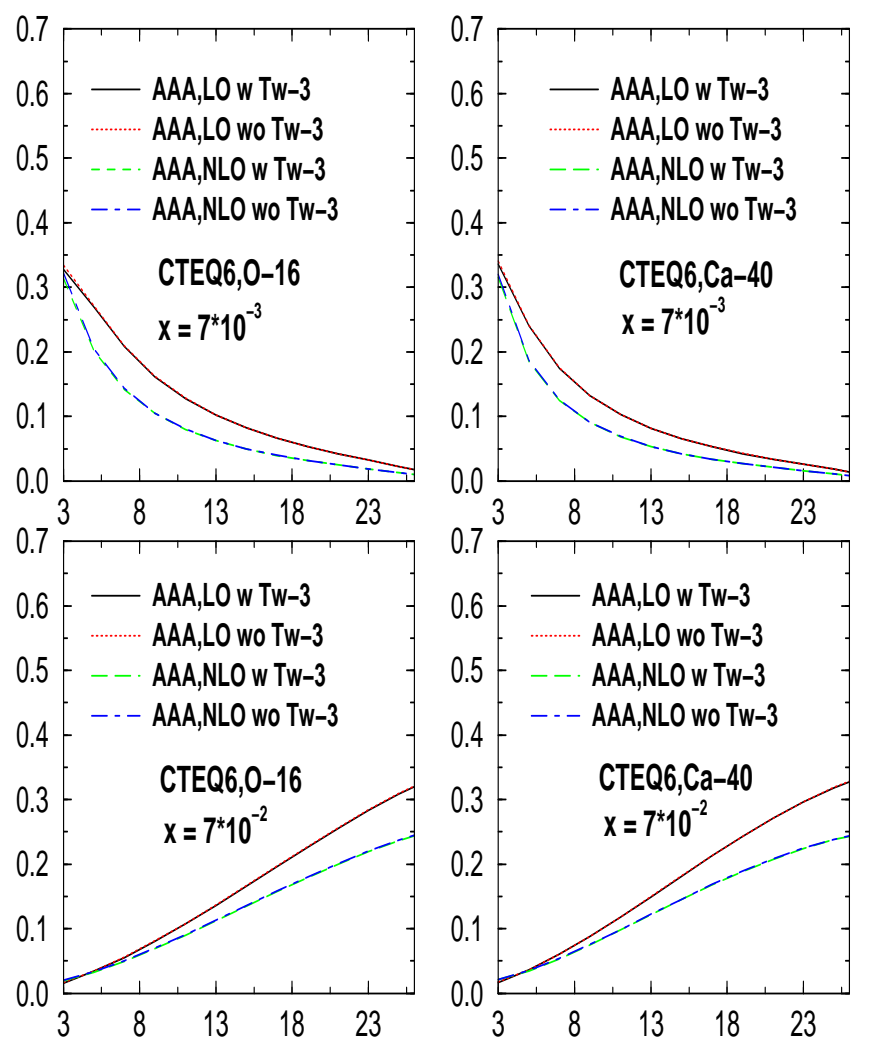

Fig. 8. $t$ integrated AAA in EIC kinematics vs. $Q^{2}$ for two typical values of $x_{b j}$ and $t_{\max }=-0.05 \mathrm{GeV}^{2}$. "W" stands for with and "WO" stands for without. 

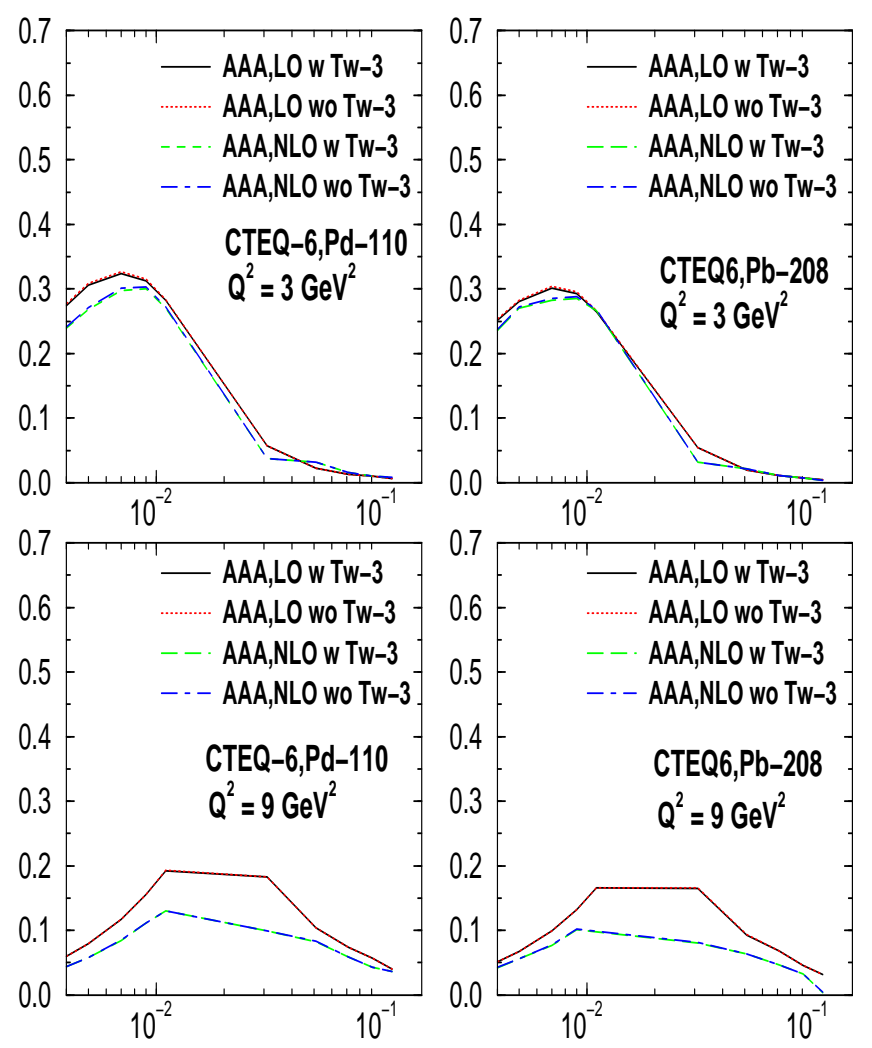

Fig. 9. $t$ integrated SSA in EIC kinematics vs. $x_{b j}$ for two typical values of $Q^{2}$ and $t_{\max }=-0.05 \mathrm{GeV}^{2}$."W" stands for with and "WO" stands for without.
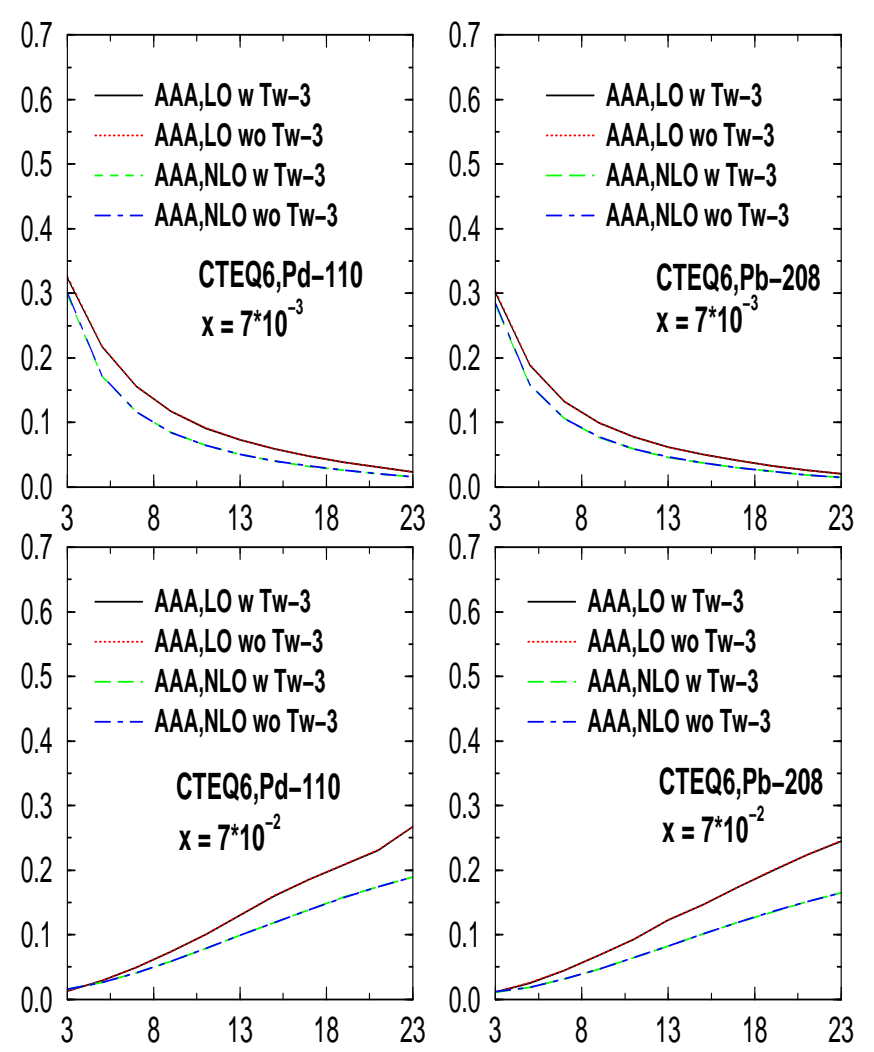

Fig. 10. $t$ integrated AAA in EIC kinematics vs. $Q^{2}$ for two typical values of $x_{b j}$ and $t_{\max }=-0.05 \mathrm{GeV}^{2}$."W" stands for with and "WO" stands for without.

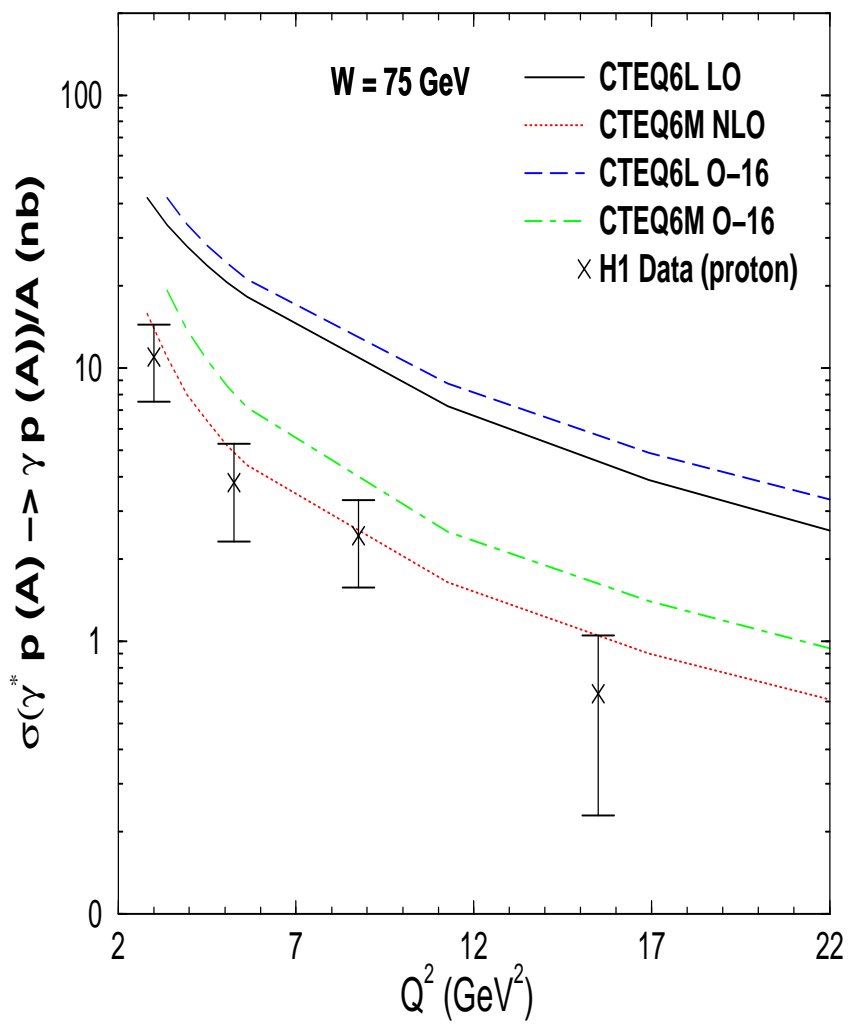

Fig. 11. One-photon cross section $\sigma\left(\gamma^{*} p\right)$ per nucleon for fixed $W$ vs. $Q^{2}$. H1 data on the nucleon is plotted in comparison within the same kinematics.

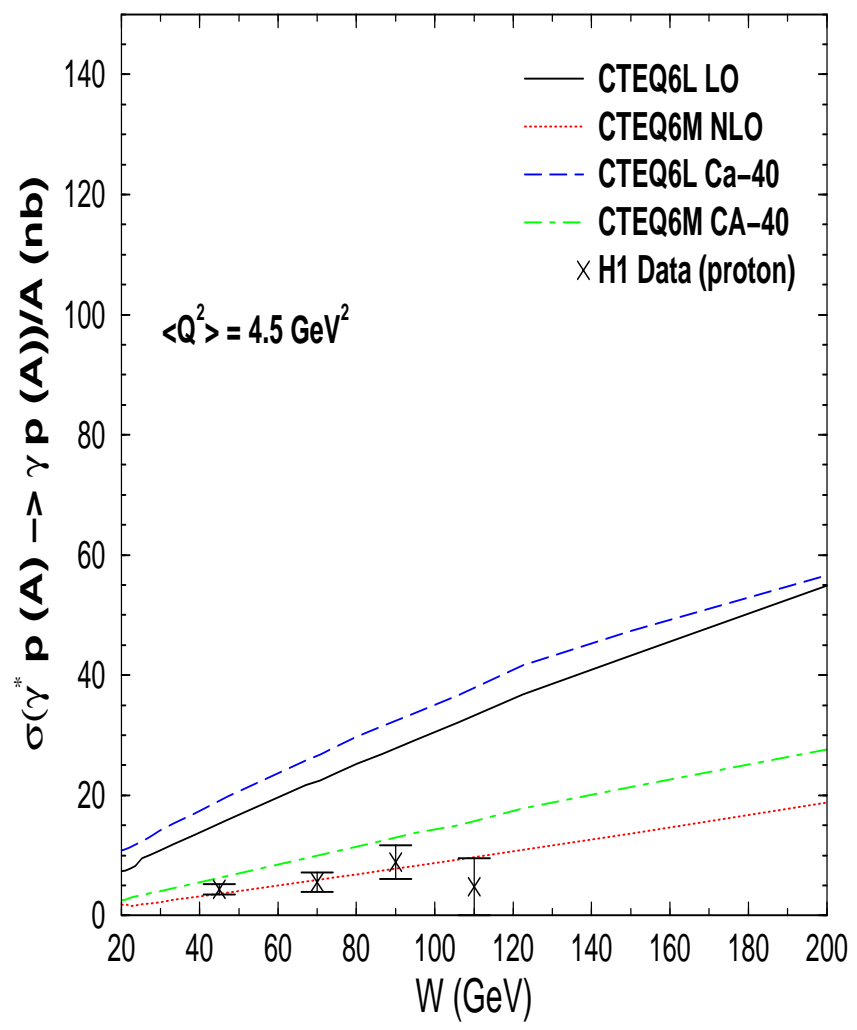

Fig. 12. One-photon cross section $\sigma\left(\gamma^{*} p\right)$ per nucleon for fixed $Q^{2}$ vs. $W$. H1 data on the nucleon is plotted in comparison within the same kinematics. 


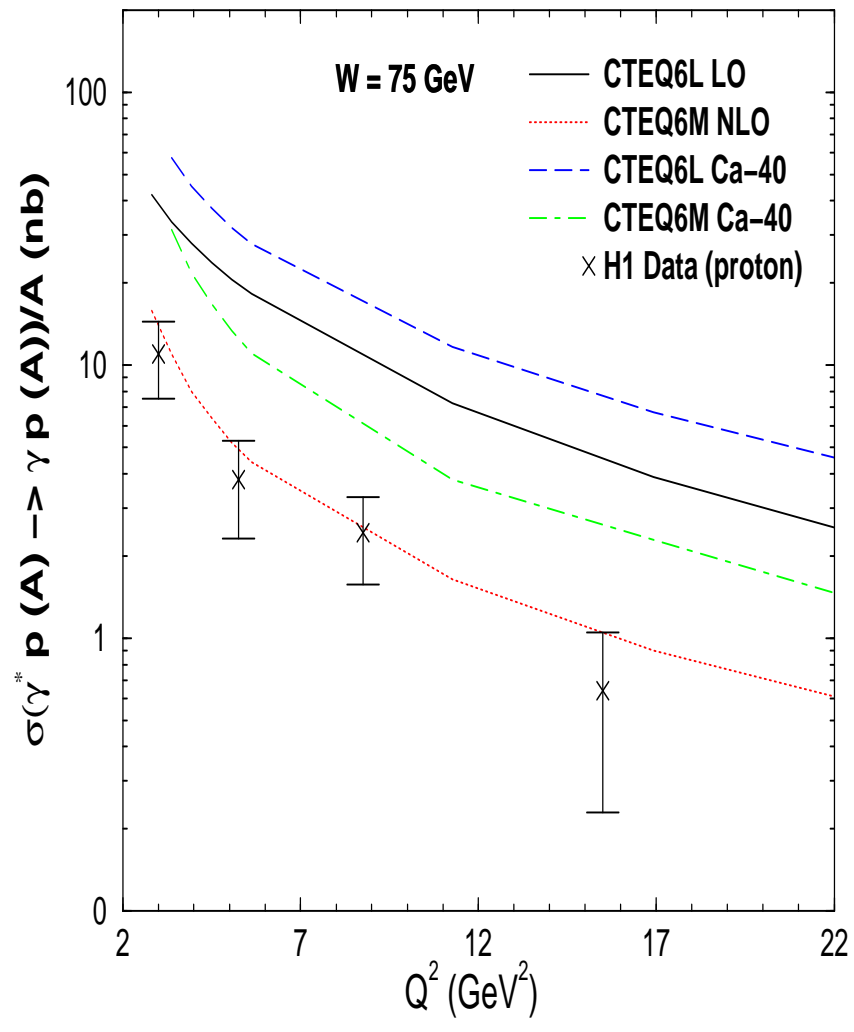

Fig. 13. One-photon cross section $\sigma\left(\gamma^{*} p\right)$ per nucleon for fixed $W$ vs. $Q^{2}$. H1 data on the nucleon is plotted in comparison within the same kinematics.

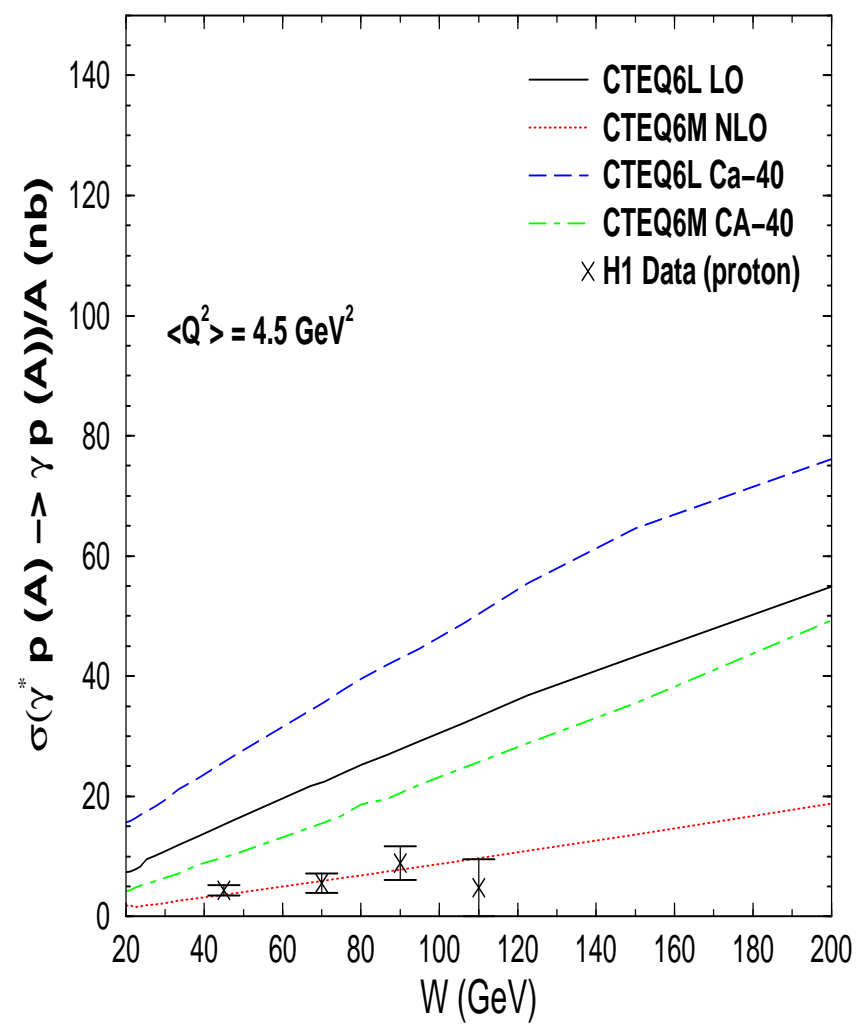

Fig. 14. One-photon cross section $\sigma\left(\gamma^{*} p\right)$ per nucleon for fixed $Q^{2}$ vs. $W$. H1 data on the nucleon is plotted in comparison within the same kinematics.

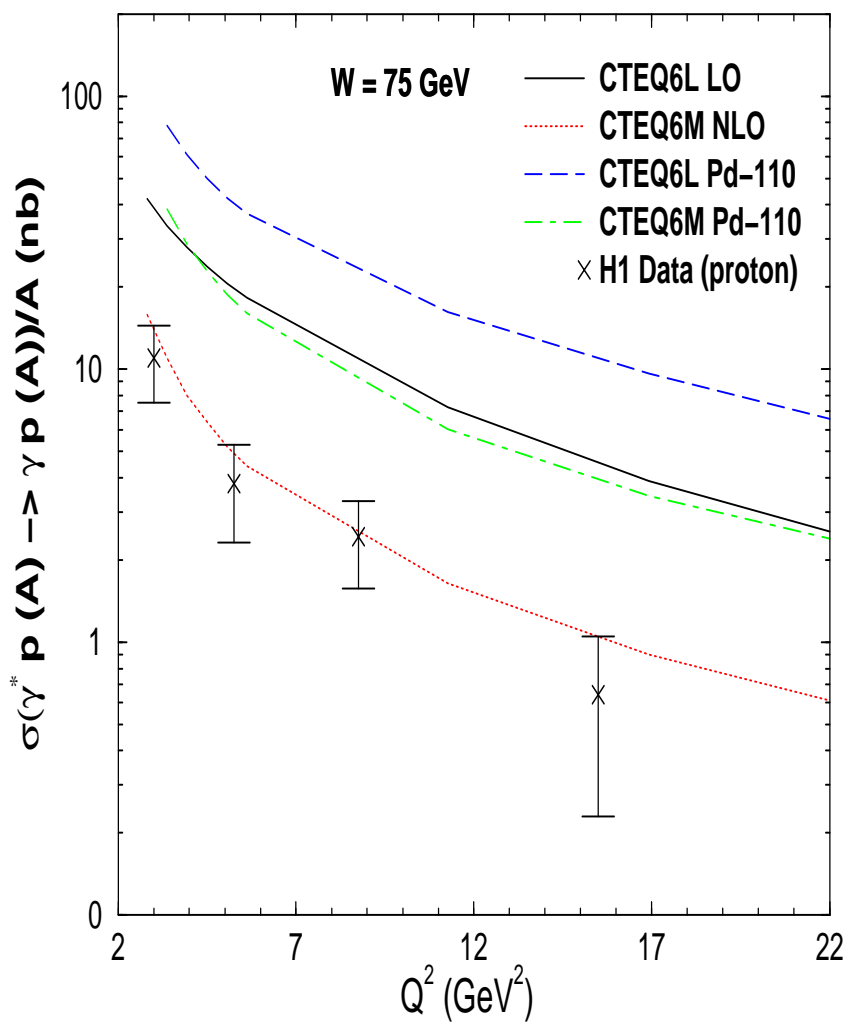

Fig. 15. One-photon cross section $\sigma\left(\gamma^{*} p\right)$ per nucleon for fixed $W$ vs. $Q^{2}$. H1 data on the nucleon is plotted in comparison within the same kinematics.

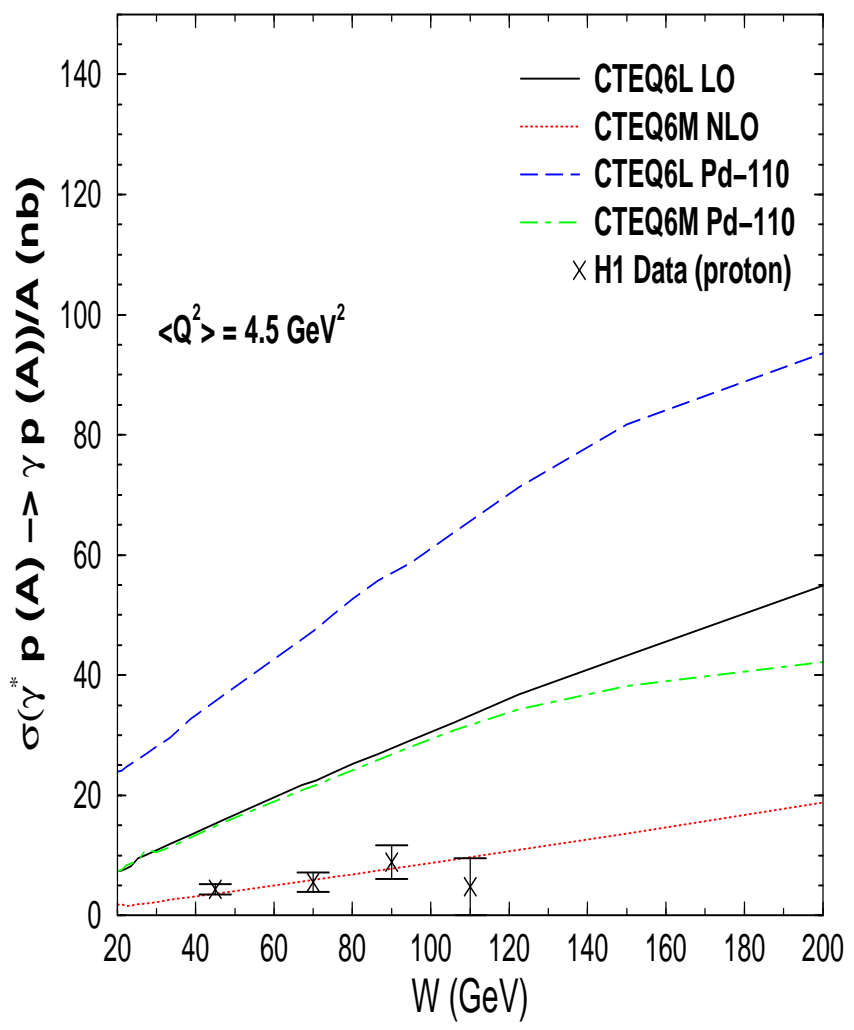

Fig. 16. One-photon cross section $\sigma\left(\gamma^{*} p\right)$ per nucleon for fixed $Q^{2}$ vs. $W$. H1 data on the nucleon is plotted in comparison within the same kinematics. 


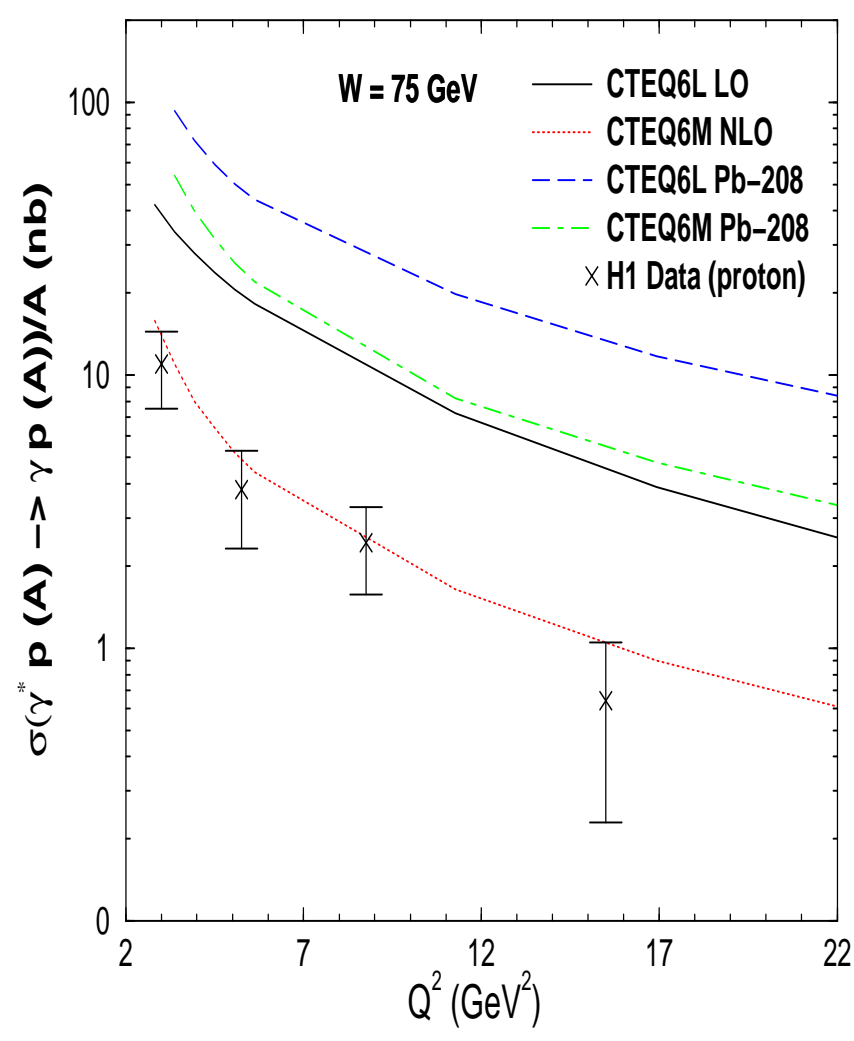

Fig. 17. One-photon cross section $\sigma\left(\gamma^{*} p\right)$ per nucleon for fixed $W$ vs. $Q^{2}$. H1 data on the nucleon is plotted in comparison within the same kinematics.

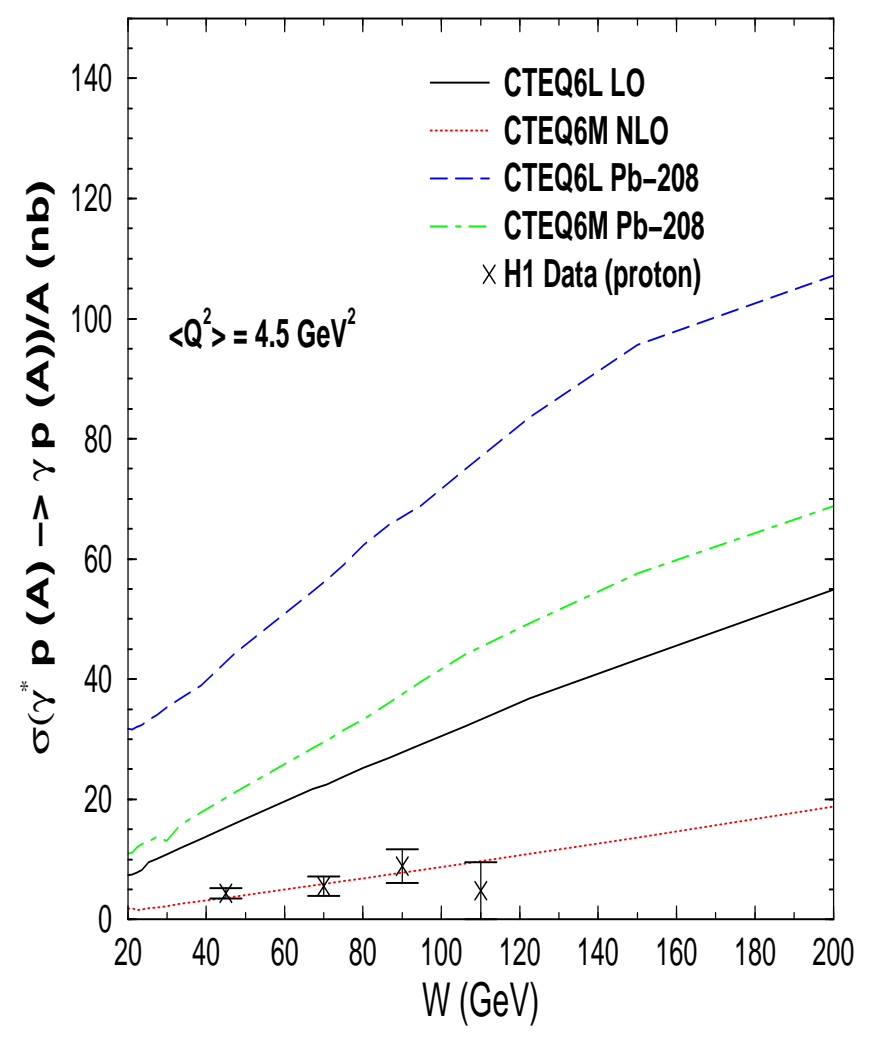

Fig. 18. One-photon cross section $\sigma\left(\gamma^{*} p\right)$ per nucleon for fixed $Q^{2}$ vs. $W$. H1 data on the nucleon is plotted in comparison within the same kinematics.
The information obtained from studies of nuclear DVCS will also enable us to investigate the expected pattern of perturbative color opacity in the coherent production of vector mesons on nuclei 21] in DIS at small $x_{b j}$, in an essentially model independent way.

\section{Acknowledgment}

We thank Vadim Guzey for providing the numerical results for the nuclear PDFs. This work was supported by the DFG under the Emmi-Noether grant FR-1524/1-3 and the DOE under grant number DE-FG02-93ER40771. MS's research was also supported by the A.v.Humbold foundation.

\section{References}

1. H1 Collaboration, C. Adloff et al., Phys. Lett. B 517, 47 (2001).

2. Zeus Collaboration, hep-ex/0305028

3. A. Freund and M. McDermott, Eur. Phys. J. C 23, 651 (2002), Phys. Rev. D 65091901 (2002).

4. A. Freund, M. McDermott and M. Strikman, Phys. Rev. D67, 036001 (2003).

5. A. Freund, Eur. Phys. J. C31, 203 (2003).

6. A. V. Belitsky, D. Müller, Nucl. Phys. A 711 (2002) 118.

7. M. Diehl, Eur. Phys. J. C25 (2002) 223.

8. X. Ji, Phys. Rev. Lett. 91, (2003) 062001, A. Belitsky, X. Ji and F. Yuan, hep-ph/030738.

9. A. Freund and M. Strikman, hep-ph/0307211

10. L. Frankfurt, V. Guzey and M. Strikman, hep-ph/0303022

11. F. Ellinghaus (for the HERMES Coll.), hep-ex/0212019

12. V. Guzey and M. Strikman, Phys.Rev. C68 (2003) 015204.

13. A. Kirchner and D. Müller, hep-ph/0302007 hep-ph/0202279

14. F. Cano and B. Pire, Nucl. Phys. A711 (2002) 133, hep-ph/0307231

15. A. Belitsky, A. Kirchner and D. Müller, Phys. Rev. D 64 116002 (2001).

16. EIC Whitepaper.

17. A. Freund, hep-ph/0306012

18. A. V. Belitsky and D. Müller, Nucl. Phys. B 589 (2000), 611, A. V. Radyushkin and C. Weiss, Phys. Lett. B 493 (2000) 332, Phys. Rev. D 63 (2001) 114012, I. V. Anikin, B. Pire and O. V. Teryaev, Phys. Rev. D 62 (2000) 071501, N. Kivel and M. V. Polyakov, Nucl. Phys. B 600, (2001) 334. 19. J. Pumplin et al., CTEQ Collaboration, J. High Energ. Phys. 0207, 012 (2002).

20. L. L. Frankfurt, A. Freund and M. Strikman, Phys. Lett. B 460, 417 (1999) arXiv:hep-ph/9806535.

21. S. J. Brodsky, L. Frankfurt, J. F. Gunion, A. H. Mueller and M. Strikman, Phys. Rev. D 50, 3134 (1994) arXiv:hep-ph/9402283. 